\title{
From Action to Language: Comparative Perspectives on Primate Tool Use, Gesture and the Evolution of Human Language
}

James Steele, Pier Francesco Ferrari, and Leonardo Fogassi (compilers and eds.)

Philosophical Transactions of the Royal Society B, Volume 367, 2012 (paperback), £59.50.

ISBN-13: 9780854039234.

\section{Reviewed by MARLIZE LOMBARD}

Department of Anthropology and Development Studies, University of Johannesburg, PO Box 524, Auckland Park, Johannesburg 2006, SOUTH AFRICA; mlombard@uj.ac.za

$\mathrm{W}$ ith rapidly growing numbers of paleontological and archaeological finds, and increasingly sophisticated techniques that allow us to examine them and unravel their potential contributions to what we know about human evolution, this is a welcome and timely volume. The compilers, editors, and authors tackle one of the most difficult and intriguing questions of our time, namely the evolution of human language. The contents cut across several disciplines in 12 mostly well-written and adequately illustrated papers. The overarching theme is aimed at examining tool use and manual gestures in primates as a window on the evolution of the human capacity for language. In the opening paper, the compiler-editors provide the historical background to the issue by suggesting that, since Darwin, it was recognized that human hands may have evolved to facilitate tool control, and that the human vocal tract evolved to facilitate spoken language. Yet, if and how these two adaptive trends are linked remains unknown. Recent developments in clinical techniques and the neurophysiological 'mirror neuron system' hypothesis, supported by new experimental work, however, indicates a fitting time to revisit the theme in a cohesive manner. The introductory paper also provides a useful summary of the research topics covered in the volume.

In the first research paper, Iriki and Taoka set the tone by placing human brain evolution, tool-use, and language against the background of a triadic (ecological, neural, cognitive) niche construction hypothesis. They suggest that extended brain functions relating to tool use and language drove fast and radical changes in the hominin ecological niche. Building their hypothesis, the reader is introduced to ecological niche construction in terms of hominin ecology structured through incorporating different classes of tools, leading to a positive feedback between new brain functions and the resulting modifications of the habitual environment. They also discuss the parietal plasticity of the brain, and the fact that the parietal cortex must be key to gaining more advanced forms of representation. Parietal 'neural niches' for processing spatial and non-spatial cognition are compared to language that is filled with spatial metaphors for abstract thoughts, and the opercular cortex is identified as 'a cradle for language' by reusing spatial processing principles. Drawing their argument together, they present the parietal cortex of the human brain as the centre of 'triadic' interactions, a site for various sensory and motor integrations and coordinated transformations, and an extension of neural axes from concrete to virtual spaces to facilitate locomotion, tool use, and language. They admit that their argument is speculative, but I found their presentation convincing and the hypothesis potentially testable with future work.

Macellini and colleagues investigate individual and social learning processes involved in the acquisition and generalization of tool use in macaques that can use several tools. They provide replicable protocols for, and report on, three separate experimental studies. These included: a) testing the macaques' ability to generalize the use of a stick as a tool on objects with different physical features; b) whether new environmental contexts would stimulate adaptations in previously learned motor strategies; and, c) whether the observation of a skilled model might facilitate tool-use learning by naïve observer monkeys. Specific outcomes are provided for each experiment, leading to the conclusion that although social factors might be important to facilitate primate interaction with potential tools, these factors are not critical for learning to use tools. It also was found that the monkeys do not have the ability to translate a visual description into the motor programs for copying its behavioral goal. At this stage, the reader may legitimately ask how this fits in with the evolution of human language? In their concluding section, that seems somewhat separated from their main text, they attempt to address the theme of the volume by proposing that the sequential organization of behavior extends beyond tool use. It is argued that although the neural mechanisms underlying human language can only directly be observed in humans, anatomical and functional data suggest that the neural substrates of sequential organization in non-human primates provided the fabric for extending the cortical motor system, ultimately facilitating speech.

The next paper is a pleasure to read, even a novice can follow the language and discussions without effort. It is well organized and the experimental and data collection protocols fully replicable. Here, magnetic resonance imaging and a behavioral study are employed by Hopkins and colleagues to trace the neural and cognitive correlates of aimed throwing in chimpanzees. They examined whether differences in the ratio of white to grey matter were evident in the homologue to Broca's area and the motor-hand area of the precentral gyrus in chimpanzees that throw reliably to those that do not. The outcome is that the proportion of white matter in Broca's homologue and the motor-hand 
area of the precentral gyrus was significantly higher in chimpanzees that throw reliably, compared to those who do not. Also, the asymmetries in white matter within both regions were larger in the hemisphere contralateral to the subject's preferred throwing hand. Chimpanzees with more reliable throwing skills showed significantly better communication skills than their less accurate counterparts. The conclusion is that chimpanzees that have learned to throw more accurately have developed greater cortical connectivity between the primary motor cortex and the Broca's area homologue, and that during hominin evolution increasing selection for well-aimed throwing in a context of hunting and/or defense potentially refined the neural architecture of the left hemisphere to eventually facilitate other complex motor sequencing actions such as those associated with modern speech and language.

Frey and Povinelli present the results of another set of experiments with chimpanzees. Their study is pitched towards testing whether chimpanzees, have the ability to adjust their ongoing actions in the anticipation of forthcoming task demands, which is considered evidence of internal action representations in the case of humans. Thus, the behaviors we choose in the present differ, depending on what we intend doing next. This tendency to 'project' points to a specialized role for mechanisms within the human cerebellum and dominant left cerebral hemisphere as representing the likely sensory costs of intended future actions. In their introductory section the authors consider several issues such as response selection versus action selection, neural substrates of response and action selection, cerebral asymmetry, context sensitivity in action selection, and evidence for predictive action selection in non-human primates. They then present the protocols and results for two 'grippreference' experiments. Their results lead to the conclusion that it is yet unknown whether the neural mechanisms involved in anticipatory planning are organized similarly across primate species. Yet, for humans there is mounting evidence that the involvement of cerebellar, cortical, and sub-cortical mechanisms in predictive functions shows a distinct left cerebral asymmetry. Whether this asymmetry is causally related to right-hand dominance and/or the evolution of language remains unknown, but predictive planning may extend further back into our primate origins than previously thought.

By juxtaposing two strands of research, Bril and colleagues identify unsolved problems that could be addressed by either experimental work on behavioral organization and skill acquisition in tool behavior, or a comparative approach to the neuropsychology of primate tool-use. To the point, they start from the archaeological perspective that stone-tool production has been basic to hominin behavior for the last 2.6 Myr, and therefore became diagnostic of the cognitive ability and motor skills of extinct hominins. They provide readers with thorough overviews of their own experiments designed to investigate the functional mastery of percussive technology in nut-cracking and stone flaking actions. Where possible, they enrich their experimental outcomes with recent hunter-gatherer ethnographies.
However, referring to the !Kung of the Kalahari as a 'quintessential' example of modern hunter-gatherers is perhaps awkward in current socio-anthropological as well as local political debate. But, admittedly, as a South African, I am hyper-sensitive regarding such issues. They also own up to the fact that the work does not directly address African ape-human cognitive and behavioral contrasts, but suggest that a model that combines comparative neuropsychology of primate tool-use with descriptive anatomical and statistical analyses of anthropoid primate brain evolution, focusing on cortico-cerebellar systems, might provide a way forward. Generating any links to the evolution of the human capacity for language is, however, left up to the reader.

Stout and Chaminade provide a stimulating review paper in which they first summarize current debate directly relating to stone tools, language, and the brain in human evolution. Similar to the first research paper in the volume, they start with Darwin, ending their introduction with reference to a recent series of relevant articles. They argue that an improved understanding of the germane structure-function relationships in modern humans is key to identifying potential evolutionary relationships between behaviors such as tool-making and speech. A useful discussion is provided, especially for non-neuroscientists, of the cortical networks supporting speech and tool-use, which are both goal-directed motor acts. Stone tool-making is then discussed in relation to brain evolution. Their focus is on the Oldowan and Acheulean traditions of the Earlier Stone Age. The first interpreted as possibly contributing to producing a 'language ready brain,' and the late stages of the second to reflect the ability of hominins to implement hierarchically complex, multi-stage action sequences. Handaxes of the late Acheulean are thus seen as evidence of cognitive control processes that are computationally and anatomically similar to some of those involved in modern human discourse-level language processing. Under the rubric of 'intentional communication,' they also review the technological pedagogy hypothesis. Importantly, they remind us that valid reasons for skepticism remain for, and substantial differences in detail exist between, the different evolutionary hypotheses about the 'gestural' and/or 'technological' origins of language.

Shifting our attention from Africa-based or -derived apes and hominins, Barney and colleagues investigate the articulatory capacity of Neanderthals as representing a very recent, human-like fossil hominin. However, it is emphasized that they do not want to contribute to the polarization and polemic that often characterize discourse about whether or not Neanderthals were 'human-like.' They start by investigating the speech, handedness, and tool-use nexus in these humans, who can be seen as our closest extinct relatives. Original research outcomes are provided for their reconstructions of the positions of the hyoid and tongue root of Neanderthals, and a software articulatory model that allows them to explore the effects on vowel space, varying the position of the hyoid and other articulators. By generating and processing data from modern humans and Neanderthal fossil material using computed tomogra- 
phy (CT) scans, human regression models can be applied to Neanderthal reconstructions for acoustic modeling - the results can be listened to in three sound files attached to the electronic supplementary material. This new approach is proposed as a way of incrementally building an understanding of, and perhaps direct evidence for, Neanderthal speech potential. This is an entertaining, yet serious, research contribution with much future potential-certainly something that will fascinate, and potentially help focus, many a graduate mind.

In their contribution, Pastra and Aloimonos emphasize the fact that language and action share a common neural basis and 'syntax' or similar hierarchical and compositional organization. They immediately place the reader in the human domain of dancing and routine household activities and ask some poignant questions of us before contextualizing their research. An astute background is provided to the hunt for the structural principles of visual and motoric action, and the relationship between language and the hierarchical structure of such action. Focus areas include neurobiological approaches on action grammar, and computational grammars of action. They situate their approach within the latest evolution of the Chomskyian tradition of generative grammars into the minimalist program. In a formal language-analysis framework, they provide jampacked discussions aided by informative schematic illustrations. Their outcomes are self-assessed by asking a series of questions that can be collapsed into whether linguistic features such as 'recursion,' 'merging,' and 'move' are all necessary for an action grammar. They explain each of these features, and suggest that the generative grammar of action must allow for recursion and the use of merging and move operators. Their main arguments, namely how structuring action has important implications for action learning and generalization in human cognition research and computation, are colorfully illustrated. It will not be an easy read for the common anthropology/archaeology student or enthusiast interested in the evolution of human language. Yet, their conclusion that action structure is recursive, and that it is tool-use that drives both merging and recursion, is an important additional strand of evidence for the link between the evolution of human language and technology. Their approach is thus noteworthy to a broad spectrum of disciplines.

Liebal and Call takes us back to non-human primate research, this time, with specific focus on manual gesturing. They explore how gestures originated out of actions before introducing the debate about whether the origins of gestures were phylogenetic or ontogenetic. All this is aimed at identifying criteria suited for distinguishing a true 'gesture' from non-communicative actions. Building on this exploration, they move onto the potential origins of human language by acknowledging the intense debate in which some researchers support a vocal origin and others hold gestures as precursors to human language. Even though there is no reconciling theory, they suggest that discovery of a mirror-neuron system for grasping in monkeys might emphasize the role of gestures because it represents the link between actions and communication. They also highlight that the relevant neurons in monkey brains are located in a region analogous to that of Broca's area in human brains. A brief overview is then provided of theories suggesting a gestural origin for human language, followed by a discussion of the lateralization of hand-use while gesturing in non-human primates. They conclude that: a) non-human primate gesturing is highly variable and different from human gestures in many aspects; $b$ ) some form of social learning might be implicated-but this needs further empirical assessment; c) although there is some evidence of laterality in captive individuals, the results are mixed for non-human primate species on population level, so that it is too early to generalize a right-handed preference for gestures in our closest relatives; and, d) that much work remains regarding tracing the origins of gestures and their relationship to the evolution of human language.

In the next review paper, the themes of action and gesturing are expanded on by drawing parallels between them and mental representation in humans and non-human primates. Cartmill and colleagues argue that the movements we make with our hands mirror and shape what and how we think. For those interested in cognitive and conceptual processes, and how these are expressed, this paper is highly recommended. It is a comfortable read in which the authors investigate relationships between action, gesture, and thought, discussing the role of these relationships in the evolution of language. Gesture is presented as a bridge between action and thought, and it is implied that gesturing has a stronger effect on the mental representation of an action, than doing the action has. The authors highlight how language-trained apes demonstrate the level of abstract cognition that apes are able to reach when stimulated within a humanized environment. This example is used to point to the importance of a rearing environment for the expansion of cognitive and communicative aptitude. In their unpacking of gesture and mental representation in the evolution of language, they too emphasize the importance of mirror-neurons, and provide the general reader with a useful introduction to the topic and its relevance to the theme at hand. In their closing section, it is suggested that manual gesturing to replicate actions and objects, while not acting on real-world objects, signifies a cognitive leap in hominid evolution. But for them, far-reaching social changes, in addition to an increased pressure for accurate tool production and use, would have been required for the development of human language, because apes (even when languagetrained and exposed to humanized conditions) do not develop the social and representational milestones that young children do.

The paper chosen to conclude the volume provides a neuropsychological perspective on the link between language and praxis (complex actions) in modern humans. Roby-Barmi and colleagues provide an extensive introduction to how language and praxis, including tool-use, are cognitive functions that are uniquely developed in humans, even though present to some degree in several non-human species. Again the mirror-neuron system is highlighted as 
an important aspect for multi-disciplinary research relating to the evolution of human language. The system is suggested to probably provide a foundation for a basic, gestural form of language, on or after which Broca's area developed as a result of ever more complex junctures in gesture recognition and imitation. Arguing that brain imaging in neuropsychological contexts now allow for the exploration of the neural bases of higher brain functions and the mechanisms of their dysfunction, the authors proceed with clinical descriptions and accounts of apasia and apraxia. Some contemporary theories for the cerebral organization of language and complex actions are presented, before a detailed discussion of how action recognition, imitation, and gestural communication are affected by aphasia and apraxia. The authors develop thoughts on gesture-imitation and speech-repetition, and on pantomime and gestural communication. They highlight how several studies show functional links between language and praxis, perhaps indicating shared processes between both cognitive abilities.
They also investigate whether language and complex actions share cerebral resources, concluding that Broca's area probably has a generic function that might be a common resource for action and language.

This is a volume chock-full of new information and updates on novel approaches. It will stimulate anyone with multi- and/or cross-disciplinary interests in the evolution of language-human or otherwise. As a unit it is thoughtprovoking and provides a range of engagement levels to readers who might be more or less knowledgeable of and/ or specialized in the different fields. A concluding paper in which the work is synthesized into an up-to-date interpretation of the evolution of human language would have been interesting, but perhaps too ambitious at this point in time. As an archaeologist who is peripherally (but admittedly increasingly) interested in the evolution of human language and cognition, I know that this volume will become a valuable resource for me and graduate students in the years to come. 